MITSUBISHI ELECTRIC RESEARCH LABORATORIES

http://www.merl.com

\title{
Co-Design of Nonlinear Control Systems with Bounded Control Inputs
}

\author{
Wang, Y.; Bortoff, S.A.
}

TR2014-068 June 29, 2014

\begin{abstract}
This paper considers co-design of nonlinear constrained control systems: simultaneous design of the nonlinear plant and control policy where the control is bounded. Similar to prior art, the co-design is attacked as a non-convex optimization problem, which is solved by using an improved policy iteration scheme. We have proved rigorously that the system performance can be improved after each step of the proposed policy iteration scheme until convergence to a sub-optimal solution is attained. Effectiveness of the proposed methodology is illustrated through the co-design of a load-positioning system.
\end{abstract}

World Congress on Intelligent Control and Automation (WCICA)

This work may not be copied or reproduced in whole or in part for any commercial purpose. Permission to copy in whole or in part without payment of fee is granted for nonprofit educational and research purposes provided that all such whole or partial copies include the following: a notice that such copying is by permission of Mitsubishi Electric Research Laboratories, Inc.; an acknowledgment of the authors and individual contributions to the work; and all applicable portions of the copyright notice. Copying, reproduction, or republishing for any other purpose shall require a license with payment of fee to Mitsubishi Electric Research Laboratories, Inc. All rights reserved. 



\title{
Co-Design of Nonlinear Control Systems with Bounded Control Inputs
}

\author{
Yebin Wang and Scott A. Bortoff \\ Mitsubishi Electric Research Laboratories \\ 201 Broadway, Cambridge, MA 02139, USA. \\ Email: yebinwang@ieee.org, bortoff@merl.com
}

\begin{abstract}
This paper considers co-design of nonlinear constrained control systems: simultaneous design of the nonlinear plant and control policy where the control is bounded. Similar to prior art, the co-design is attacked as a non-convex optimization problem, which is solved by using an improved policy iteration scheme. We have proved rigorously that the system performance can be improved after each step of the proposed policy iteration scheme until convergence to a sub-optimal solution is attained. Effectiveness of the proposed methodology is illustrated through the co-design of a load-positioning system.
\end{abstract}

Keywords-Nonlinear systems, Co-design, Constrained optimal control, Policy iteration.

\section{INTRODUCTION}

The plant and the control design of nonlinear control systems is typically decoupled, i.e. the plant, also referred to as the open-loop system, is given a priori while designing the control policy. Such decoupling philosophy is popular in practice albeit not necessary since both the plant and the control jointly affect the closed-loop system performance. Slight adjustments of the plant may result in remarkable improvements of the system performance. Here, by "co-design", we refer to the simultaneous design of both the plant and the control policy to optimize prescribed performance objectives. Similar research work has been conducted under the names of "integrated structure and control design" [1], [2], "optimal redesign" [3], [4], and "simultaneous design" [5], [6], etc. The co-design problem can find a great number of engineering applications, such as the optimal design and control for aerospace crafts [5], [6], smart buildings [2], [4], and electromechanical devices [7].

One commonly used approach to the co-design problem is to formulate it as a nonlinear optimization problem, which may commonly be non-convex [3], by parameterizing the open-loop system as well as the control policy. The resultant optimization problem is challenging due to the non-convexity. When nonlinear system dynamics and non-quadratic cost functions are taken into consideration, there is less hope of solving the problem. Even for fixed system parameters, finding the optimal control policy requires solving the well-known Hamilton-Jacobi-Bellman (HJB) equation of which a closedform solution is not practical to be obtained in general cases.

With the optimization formulation of the co-design problem, some work has been devoted to establish the existence and uniqueness of an optimal solution [8], and most of existing work assume the existence of optimal solutions and study the mathematical characterization of an optimal solution or methods to solve for a sub-optimal solution [9]-[12]. This paper focuses on new methods to solve nonlinear co-design problems which are naturally resulted from co-design of nonlinear constrained control systems. This paper extends work [2], [3], [13], [14] in the following two-folds: nonlinear system dynamics and non-quadratic cost functions are considered; control input is constrained. The main idea is to modify the conventional policy iteration technique [15]-[17], by adding an extra optimal system design step to update the system parameters. We show the system performance can be improved until the convergence to a sub-optimal solution.

The remainder of the paper is organized as follows. Section II formulates the nonlinear co-design problem. Section III presents the modified policy iteration scheme. Section IV validates the proposed method by its application to a loadpositioning system. Section V concludes this paper.

\section{PROBLEM FORMULATION}

Consider the following continuous-time control-affine nonlinear system

$$
\dot{x}=f(x, \theta)+g(x, \theta) u, \quad x(0)=x_{0},
$$

where $x \in \mathbb{R}^{n}$ is the state, $u \in \mathbb{R}^{m}$ is the control input, and $\theta \in \mathbb{R}^{l}$ is the vector of designable system parameters. The control $u$ is lower- and upper-bounded element-wisely by constant vectors $u_{\min } \in \mathbb{R}^{m}$ and $u_{\max } \in \mathbb{R}^{m}$, respectively; the system parameters are lower- and upper-bounded component-wisely by $\theta_{\min } \in \mathbb{R}^{l}$ and $\theta_{\max } \in \mathbb{R}^{l}$, respectively. For simplicity of notation, we denote the constraints on $u$ as $u_{\min } \leq u \leq u_{\max }$ and the constraints on $\theta$ as $\theta_{\min } \leq \theta \leq \theta_{\max }$. The vector fields $f: \mathbb{R}^{n} \times \mathbb{R}^{l} \rightarrow \mathbb{R}^{n}$ and $g: \mathbb{R}^{n} \times \mathbb{R}^{l} \rightarrow \mathbb{R}^{n \times m}$ are locally Lipschitz satisfying $f(0, \theta)=0$, for $\theta_{\text {min }} \leq \theta \leq \theta_{\max }$. The cost for the co-design problem to minimize is inspired from [18] and takes the following expression

$$
J\left(x_{0}, \theta, u\right)=\int_{0}^{\infty}[Q(x)+L(u)] d t,
$$

where $Q(x)$ is a positive definite function, and

$$
\begin{aligned}
L(u) & =2 \int_{0}^{u}\left(\Phi^{-1}(v)\right)^{T} R d v, \\
\Phi(v) & =\left[\phi_{1}(v), \cdots, \phi_{m}(v)\right]^{T}, \\
\Phi^{-1}(u) & =\left[\phi_{1}^{-1}(u), \cdots, \phi_{m}^{-1}(u)\right]^{T},
\end{aligned}
$$

with $R$ a symmetric positive definite matrix, and $\Phi(v)$ a bounded smooth one-to-one, and monotonic odd function satisfying $\Phi(0)=0$ and $u_{\min } \leq \Phi(v) \leq u_{\max }, \forall v \in \mathbb{R}^{m}$. 
Definition 1. Let $\Omega$ be a compact set containing the origin in its interior. Consider system (1) and the cost (2). A feedback control policy $u(x)$ is called admissible with respect to the vector of parameters $\theta$, if

1) $\quad u_{\min } \leq u(x) \leq u_{\max }, \forall x \in \Omega$;

2) the closed-loop system composed of (1) and $u(x)$ is asymptotically stable on $\Omega$;

3) $J\left(x_{0}, \theta, u\right)$ is finite, if $x(t) \in \Omega, \forall t \geq 0$.

Denote $U_{\theta}$ as the set of all admissible control policies corresponding to $\theta$ satisfying $\theta_{\min } \leq \theta \leq \theta_{\max }$. We introduce the following assumption on the system (1).

Assumption 1. There exists $\theta_{0}$ and $u_{0}(x)$ satisfying $\theta_{\min } \leq$ $\theta_{0} \leq \theta_{\max }$, such that $u_{0} \in U_{\theta_{0}}$.

Given any $\theta$ satisfying $\theta_{\min } \leq \theta \leq \theta_{\max }$ and an admissible control $u(x) \in U_{\theta}$, we call $(\theta, u(x))$ an admissible pair. Given $u(x) \in U_{\theta}$, the associated cost must satisfy

$$
J\left(x_{0}, \theta, u\right)=V\left(x_{0}\right),
$$

where $V(x)$ is the solution of the following partial differential equation

$$
\begin{aligned}
0= & \nabla V^{T}(x)[f(x, \theta)+g(x, \theta) u(x)] \\
& +Q(x)+L(u(x)), x \in \Omega, V(0)=0 .
\end{aligned}
$$

Remark 1. The co-design problem of the system parameters $\theta$ and the control $u(x)$ can be formulated as follows: Given system (1), find an admissible pair $\left(\theta^{*}, u^{*}\right)$ which minimizes the cost (2). As pointed out in [17], if $\theta$ is fixed, the co-design problem is reduced to a constrained optimal control problem, which can be attacked by solving the following HamiltonJacobi-Bellman (HJB) equation:

$$
\begin{aligned}
& \nabla\left(V^{*}\right)^{T}\left(f-g \Phi\left(\frac{1}{2} R^{-1} g^{T} \nabla V^{*}\right)\right)+Q \\
& +2 \int^{-\Phi\left(\frac{1}{2} R^{-1} g^{T} \nabla V^{*}\right)}\left(\Phi^{-1}(v)\right)^{T} R d v=0
\end{aligned}
$$

with $V^{*}(0)=0$, and the optimal control is given by $u^{*}=$ $-\Phi\left(\frac{1}{2} R^{-1} g^{T} \nabla V^{*}\right)$.

Remark 2. Although work [2] deals with the co-design of linear unconstrained control systems, its methodology can be readily extended to the co-design of nonlinear constrained control system (1). That is: an optimal control policy $u$ is solved from the HJB for fixed $\theta$, then $\theta$ and the control $u$ are updated by solving an optimization problem subject to a system-equivalence constraint.

Since the HJB is difficult to solve, our intention is to circumvent the HJB by formulating the co-design problem as follows.

Problem 1 (Co-design problem).

$$
\begin{aligned}
& \min _{\theta, V, u,} J\left(x_{0}, \theta, u\right)=V\left(x_{0}\right) \\
& \text { s.t. } \text { 1) } 0=\nabla V^{T}(x)[f(x, \theta)+g(x, \theta) u(x)] \\
&+Q(x)+L(u(x)), \forall x \in \Omega, \\
& \text { 2) } 0=V(0), \\
& \text { 3) } u \in U_{\theta}, \\
& \text { 4) } \theta_{\min } \leq \theta \leq \theta_{\max } .
\end{aligned}
$$

Remark 3. As it will become clear in the sequel, introducing the constraint (4) instead of the HJB in Problem 1 enjoys several advantages: the constraint (4) is relatively simpler than the HJB in the sense that it might be easier to handle by convexification techniques; the constraint (4) is much easier to solve than the HJB which is a nonlinear partial differential equation; the constraint allows us to reuse the well-established policy iteration idea for optimal control design.

Problem 1 is difficult to solve for at least two reasons. First, this optimization problem is generally non-convex and solving non-convex constrained optimization problems are not only computationally expensive, but also have no guaranteed convergence to an optimal solution. Second, nonlinearities involved in the problem make it almost impossible to find an analytic solution even for a fixed $\theta$.

\section{AN ITERATIVE TECHNIQUE FOR SOLVING THE CO-DESIGN PROBLEM}

We first study an optimal system design problem where system parameters are decision variables with control fixed, then present an iterative algorithm for solving Problem 1.

\section{A. Optimal system design}

We begin with an admissible pair $\left(\theta_{i}, u_{i}\right)$ where $\theta_{\min } \leq$ $\theta_{i} \leq \theta_{\max }$ and $u_{i} \in U_{\theta_{i}}$. Our purpose is to find a new vector of system parameters $\theta_{i+1}$, such that the cost can be minimized. The resultant optimal system design problem can be formulated as follows:

Problem 2 (Optimal system design problem).

$$
\begin{aligned}
&\left(\theta_{i+1}, S_{i}\right)= \arg \min _{\theta, S} S\left(x_{0}\right) \\
&\text { s.t. } 1) \quad 0= \nabla S^{T}(x)\left[f(x, \theta)+g(x, \theta) u_{i}(x)\right] \\
& \quad+Q(x)+L\left(u_{i}(x)\right), \quad x \in \Omega \\
& \\
& \text { 2) } \quad S(x) \geq 0, \forall x \in \Omega \text { and } S(0)=0 \\
& \text { 3) } \quad \theta_{\min } \leq \theta \leq \theta_{\max } .
\end{aligned}
$$

Technical challenges of solving Problem 2 come from the equality constraint 1) and non-convexity of constraints 1)-2). A standard technique to handle an equality constraint is to relax it into an inequality constraint. We thus have the following relaxed optimal system design problem.

Problem 3 (Relaxed optimal system design problem).

$$
\begin{aligned}
& \left(\bar{\theta}_{i+1}, \bar{S}_{i}\right)=\arg \min _{\bar{\theta}, \bar{S}} \bar{S}\left(x_{0}\right) \\
& \text { s.t. } 1) \quad 0 \geq \nabla \bar{S}^{T}(x)\left[f(x, \bar{\theta})+g(x, \bar{\theta}) u_{i}(x)\right] \\
& +Q(x)+L\left(u_{i}(x)\right), \quad x \in \Omega, \\
& \text { 2) } \bar{S}(x) \geq 0, \forall x \in \Omega \text { and } \bar{S}(0)=0 \text {, } \\
& \text { 3) } \theta_{\min } \leq \bar{\theta} \leq \theta_{\max } \text {. }
\end{aligned}
$$

The optimal solution of a relaxed problem is generally different from its original problem. When looking into Problems 2 and 3 , one can readily show that both problems have the same optimal solutions. This is because the equality constraint (5) essentially represents necessary optimality conditions, thus defines a subset $\mathcal{O}$ containing all optimal solutions. The optimal 
solutions of Problem 3 which has a larger feasible domain than Problem 2, still belong to the subset $\mathcal{O}$.

Remark 4. After replacing the equality sign in (5) with an inequality sign, we derive a set of sufficient conditions which ensure the satisfaction of non-convex inequality constraints (6). Under certain conditions, the sufficient conditions are convex thus the non-convex inequality constraint (6) is relaxed to convex inequality constraints. Schur complement condition is applied to derive the sufficient conditions [19].

Define $V(x)$, with $V(0)=0$, as the solution of

$$
0=\nabla V_{i}^{T}(x) f_{i}(x)+Q(x)+L\left(u_{i}(x)\right), \quad \forall x \in \Omega,
$$

where $f_{i}(x)=f\left(x, \theta_{i}\right)+g\left(x, \theta_{i}\right) u_{i}(x)$. Denote

$$
\begin{aligned}
\delta f_{i}(x, \bar{\theta}) & =f(x, \bar{\theta})-f\left(x, \theta_{i}\right)+\left[g(x, \bar{\theta})-g\left(x, \theta_{i}\right)\right] u_{i}(x), \\
\delta V_{i}(x) & =\bar{S}(x)-V_{i}(x) .
\end{aligned}
$$

The following lemma gives sufficient conditions for (6).

Lemma 1. Given $u_{i} \in U_{\theta_{i}}$, the constraint (6) is satisfied for $\bar{\theta}$ and $\bar{S}(x)=V_{i}(x)+\delta V_{i}(x)$ if for any $x \in \Omega$, the following inequality holds

$$
\left[\begin{array}{ccc}
M(x, \bar{\theta}) & \nabla\left(\delta V_{i}(x)\right)^{T} & \left(\delta f_{i}(x, \bar{\theta})\right)^{T} \\
\nabla\left(\delta V_{i}(x)\right) & 4 \gamma^{-1} & 0 \\
\delta f_{c}(x, \bar{\theta}) & 0 & \gamma
\end{array}\right] \geq 0,
$$

where $\gamma>0$, and

$$
M(x, \bar{\theta})=-\nabla\left(\delta V_{i}(x)\right)^{T} f_{i}(x)-\nabla V_{i}^{T}(x) \delta f_{i}(x, \bar{\theta}) .
$$

Proof: For notation simplicity, in the proof we drop the argument $x$ from $\delta V_{i}(x), f_{i}(x), Q(x)$, and $u_{i}(x)$, and the arguments $(x, \bar{\theta})$ from $\delta f_{i}(x, \bar{\theta})$. Then, by Schur complement condition, the inequality (7) is equivalent to the following inequality $\forall x \in \Omega$

$$
\nabla \delta V_{i}^{T} f_{i}+\nabla V_{i}^{T} \delta f_{i}+\frac{\gamma}{4}\left|\nabla \delta V_{i}\right|^{2}+\frac{1}{\gamma}\left|\delta f_{i}\right|^{2} \leq 0, \forall x \in \Omega
$$

On the other hand, from (6) and (8) we have

$$
\begin{aligned}
& \nabla \bar{S}^{T}\left[f(x, \bar{\theta})+g(x, \bar{\theta}) u_{i}\right]+Q+L\left(u_{i}\right) \\
= & \left(\nabla V_{i}+\nabla \delta V_{i}\right)^{T}\left(f_{i}+\delta f_{i}\right)+Q+L\left(u_{i}\right) \\
= & \left(\nabla V_{i}+\nabla \delta V_{i}\right)^{T}\left(f_{i}+\delta f_{i}\right)-\nabla V_{i}^{T} f_{i} \\
= & \nabla V_{i}^{T} \delta f_{i}+\nabla \delta V_{i}^{T} f_{i}+\left(\nabla \delta V_{i}\right)^{T} \delta f_{i} \\
\leq & \nabla V_{i}^{T} \delta f_{i}+\nabla \delta V_{i}^{T} f_{i}+\frac{\gamma}{4}\left|\nabla \delta V_{i}\right|^{2}+\frac{1}{\gamma}\left|\delta f_{i}\right|^{2} \\
\leq & 0 .
\end{aligned}
$$

The proof is thus complete.

Remark 5. Since (7) is sufficient to guarantee (6), it introduces some conservativeness. The extent of the conservativeness, which is ideally minimized, is not only affected by the constraint (6) itself, but also controlled by the choice of $\gamma$. It is however not obvious to quantify the conservativeness result from $\gamma$ thus difficult to choose $\gamma$ with guaranteed performance.

Remark 6. With $\delta V_{i}$ and $\delta f_{i}$ treated as decision variables, the inequality (7) is convex for any given $x$. Further, if $f(x, \theta)$ and $g(x, \theta)$ are linearly parameterized by $\theta$, the inequality (7) is convex in $\delta \theta$ or equivalently $\theta$.
B. An iterative technique for solving the co-design problem

Assuming the vector fields $f$ and $g$ have linear parameterizations of $\theta$, we propose the following iterative algorithm.

\section{1) Initialization}

Select $u_{0}$ and $\theta_{0}$ satisfying $\theta_{\min } \leq \theta_{0} \leq \theta_{\max }$ and $u_{0} \in$ $U_{\theta_{0}}$. Set $i=0$.

\section{2) Policy evaluation}

Solve $V_{i}(x)$, with $V_{i}(0)=0$, from

$$
\begin{aligned}
0= & \nabla V_{i}^{T}(x)\left[f\left(x, \theta_{i}\right)+g\left(x, \theta_{i}\right) u_{i}(x)\right] \\
& +Q(x)+L\left(u_{i}(x)\right), \quad \forall x \in \Omega .
\end{aligned}
$$

3) Optimal system design

$$
\begin{gathered}
\left(\theta_{i+1}, \delta V_{i}\right)=\arg \min _{\theta, \delta V} \delta V\left(x_{0}\right) \\
\text { s.t. }\left[\begin{array}{ccc}
M(x, \theta) & \nabla(\delta V(x))^{T}\left(\delta f_{i}(x, \theta)\right)^{T} \\
\nabla(\delta V(x)) & 4 \gamma^{-1} & 0 \\
\delta f_{i}(x, \theta) & 0 & \gamma
\end{array}\right] \geq 0, \\
\delta V(x)+V_{i}(x) \geq 0 \text { and } \delta V(0)=0, \\
\theta_{\min } \leq \theta \leq \theta_{\max },
\end{gathered}
$$

where $\delta f_{i}=\left[f(x, \theta)+g(x, \theta) u_{i}(x)\right] \delta \theta$.

4) Policy improvement

$$
\begin{aligned}
& u_{i+1}(x) \\
& =-\Phi\left(\frac{R^{-1} g^{T}\left(x, \theta_{i+1}\right)\left(\nabla V_{i}(x)+\nabla \delta V_{i}(x)\right)}{2}\right) .
\end{aligned}
$$

\section{5) Iteration}

Repeat steps 2)-4) until the sequence $\left\{V_{i}\left(x_{0}\right)\right\}$ converges.

Theorem 1. For $i=0,1,2, \cdots$, the aforementioned algorithm has the following properties

1) $u_{i} \in U_{\theta_{i}}$;

2) $0 \leq V_{i+1}\left(x_{0}\right) \leq V_{i}\left(x_{0}\right)+\delta V_{i}\left(x_{0}\right) \leq V_{i}\left(x_{0}\right)$;

3) There exists $J^{*}>0$, such that $\lim _{i \rightarrow \infty} V_{i}\left(x_{0}\right)=J^{*}$.

Before giving the proof of Theorem 1, we first recall the following facts: $V_{i}$ is a solution of

$$
0=\nabla V_{i}^{T}(x) f_{i}(x)+Q(x)+L\left(u_{i}(x)\right), x \in \Omega, V_{i}(0)=0,
$$

where the pair $\left(\theta_{i}, u_{i}(x)\right)$ is given; $\bar{S}_{i}=V_{i}+\delta V_{i}$ is a solution of (9) thus satisfies

$$
\begin{aligned}
0 \geq & \nabla \bar{S}_{i}^{T}(x)\left[f\left(x, \theta_{i+1}\right)+g\left(x, \theta_{i+1}\right) u_{i}(x)\right] \\
& +Q(x)+L\left(u_{i}(x)\right), x \in \Omega, \bar{S}_{i}(0)=0,
\end{aligned}
$$

where the pair $\left(\theta_{i+1}, u_{i}\right)$ is given; $V_{i+1}$ is a solution of

$$
\begin{aligned}
0= & \nabla V_{i+1}^{T}(x)\left[f\left(x, \theta_{i+1}\right)+g\left(x, \theta_{i+1}\right) u_{i+1}(x)\right] \\
& +Q(x)+L\left(u_{i+1}(x)\right), x \in \Omega, V_{i+1}(0)=0,
\end{aligned}
$$

where the pair $\left(\theta_{i+1}, u_{i+1}\right)$ is given and $u_{i+1}$ is updated according to the policy improvement.

Proof: We use induction to prove 1)-2). i) The initialization of the algorithm ensures that $u_{i} \in U_{\theta_{i}}$ for $i=0$. ii) Suppose $u_{i} \in U_{\theta_{i}}$, we need to show $u_{i+1} \in U_{\theta_{i+1}}$. 


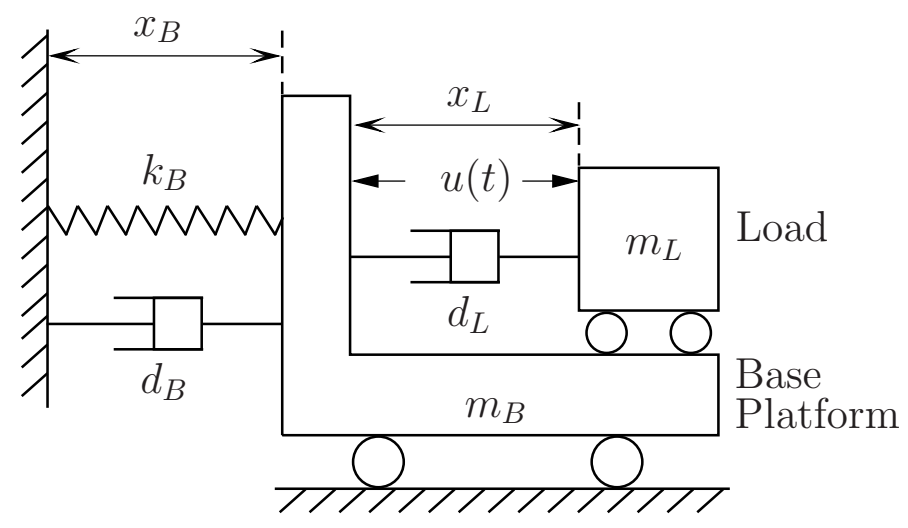

Fig. 1. Lumped parameter model of a motor-driven linear ballscrew stage supported on a flexible base platform bolted to the ground.

Since $\delta V_{i}(x)$ is the optimal solution of Problem (9), along the trajectories of (1) with $u=u_{i}(x)$, by Lemma 1 we have

$$
\bar{S}_{i}\left(x_{0}\right)=V_{i}\left(x_{0}\right)+\delta V_{i}\left(x_{0}\right) \leq V_{i}\left(x_{0}\right)
$$

Let $\hat{S}_{i}(x)$ be a positive definite function satisfying

$$
\begin{aligned}
0= & \nabla \hat{S}_{i}^{T}(x)\left[f\left(x, \theta_{i+1}\right)+g\left(x, \theta_{i+1}\right) u_{i}(x)\right] \\
& +Q(x)+L\left(u_{i}(x)\right), \quad \forall x \in \Omega, \hat{S}_{i}(0)=0 .
\end{aligned}
$$

We know $\hat{S}_{i}(x) \leq \bar{S}_{i}(x), \forall x \in \Omega$ and $x \neq 0$. Notice that (13) and (10) can be viewed as one iteration step described in [17]. Therefore, by [17, Lem. 1], it follows that

$$
V^{*}(x) \leq V_{i+1}(x) \leq \hat{S}_{i}(x) \leq \bar{S}_{i}(x), \quad \forall x \in \Omega
$$

for $i \geq 0$. Combining (12) and (14) yields 2).

Given $V_{i+1}(x)$ an finite solution of (11), we have

$$
\dot{V}_{i+1}=-Q(x)-L\left(u_{i+1}(x)\right) .
$$

Thus the closed-loop system with $u_{i+1}$ is stable. Also if $x_{0}$ is such that $x(t) \in \Omega, \forall t \geq 0$, it follows that

$$
0 \leq J\left(x_{0}, \theta_{i+1}, u_{i+1}\right) \leq V_{i}\left(x_{0}\right)+\delta V_{i}\left(x_{0}\right) \leq V_{i}\left(x_{0}\right),
$$

i.e. $J\left(x_{0}, \theta_{i+1}, u_{i+1}\right)$ is finite. We conclude that $u_{i+1} \in U_{\theta_{i+1}}$.

Since the sequence $\left\{V_{i}\left(x_{0}\right)\right\}$ is non-negative and monotonically decreasing, its limit exists. 3 ) is shown.

\section{A Numerical ExAmple}

In this section, we applied the proposed co-design method to a load-positioning system as shown in Figure 1. Its dynamics can be described by the following continuous-time linear timeinvariant system [20]

$$
\begin{aligned}
& \ddot{x}_{L}=\left(u-d_{L} \dot{x}_{L}\right)\left(\frac{1}{m_{L}}+\frac{1}{m_{B}}\right)+\frac{k_{B}}{m_{B}} x_{B}+\frac{d_{B}}{m_{B}} \dot{x}_{B}, \\
& \ddot{x}_{B}=\left(d_{L} \dot{x}_{L}-u\right) \frac{1}{m_{B}}-\frac{k_{B}}{m_{B}} x_{B}-\frac{d_{B}}{m_{B}} \dot{x}_{B},
\end{aligned}
$$

where $x_{L}$ denotes the relative displacement of the load with respect to the platform, and $x_{B}$ denotes the displacement of the platform. $d_{L}, m_{B}, m_{L}, k_{B}$, and $d_{B}$ are constant system parameters. In addition the control input is required to satisfy $-5 \leq u \leq 5$.
The co-design process is to optimize the system performance in tracking a step command. For this purpose, we define $x_{1}=x_{L}-y_{d}$, with $y_{d}$ the desired constant output, $x_{2}=\dot{x}_{L}$, $x_{3}=x_{B}$, and $x_{4}=\dot{x}_{B}$. Then the system is converted to

$$
\dot{x}=A x+B u,
$$

where $x=\left[x_{1}, x_{2}, x_{3}, x_{4}\right]^{T}$, and

$$
A=\left[\begin{array}{cccc}
0 & 1 & 0 & 0 \\
0-\frac{d_{L}}{m_{L}}-\frac{d_{L}}{m_{B}} & \frac{k_{B}}{m_{B}} & \frac{d_{B}}{m_{B}} \\
0 & 0 & 0 & 1 \\
0 & \frac{d_{L}}{m_{B}} & -\frac{k_{B}}{m_{B}}-\frac{d_{B}}{m_{B}}
\end{array}\right], B=\left[\begin{array}{c}
0 \\
\frac{1}{m_{L}}+\frac{1}{m_{B}} \\
0 \\
-\frac{1}{m_{B}}
\end{array}\right] .
$$

The cost to be minimized is chosen as

$$
J(\theta, u)=\int_{0}^{\infty}\left(100 x_{1}^{2}+0.5 \int_{0}^{v} \tanh ^{-1}(v / 5) d v\right) d t
$$

where $\theta=\left[\frac{1}{m_{L}}, \frac{1}{m_{B}}, \frac{k_{B}}{m_{B}}, \frac{d_{B}}{m_{B}}\right]^{T}$. The lower bounds, upper bounds, and initial values are shown in the second to fourth columns in Table I.

TABLE I. SYSTEM PARAMETERS

\begin{tabular}{|c|c|c|c|c|}
\hline Parameters & Min & Max & Initial & Optimized \\
\hline$\frac{1}{m_{L}}$ & 0.2 & 0.3333 & 0.2 & 1 \\
\hline$\frac{1}{m_{B}}$ & 0.04 & 0.0667 & 0.05 & 0.0667 \\
\hline$\frac{k_{B}}{m_{B}}$ & 0.4 & 1.3333 & 0.75 & 0.7496 \\
\hline$\frac{d_{B}}{m_{B}}$ & 0.004 & 0.0667 & 0.025 & 0.0041 \\
\hline
\end{tabular}

We set $\Omega=\left\{x \mid-1.2 \leq x_{1} \leq 1.2,-2 \leq x_{2} \leq\right.$ $\left.2,-1 \leq x_{3} \leq 1,-1 \leq x_{4} \leq 1\right\}$. To implement the proposed algorithm, we approximate the cost function $V_{i}(x)$ by $V_{i}(x)=\sum_{j=1}^{N} w_{i, j} \sigma_{j}(x)+\epsilon$, with $\left\{\sigma_{j}(x)\right\}$ a set of polynomials of $x$, and $\epsilon$ the approximation error. For the purpose of solving the optimization problem (9), we take 81 points on $\Omega$ and replace the constraint (9b) with a semipositive constraint of dimensions 729 , and replace $(9 \mathrm{c})$ with 81 inequalities.

The initial condition is set to be $x_{0}=[-1,0,0,0]^{T}$, and the initial stabilizing control policy is chosen as the optimal control policy with respect to the initial system parameters. The initial cost is $J=217.2996$. We execute the algorithm for 30 iterations. The optimized parameters are shown in the fourth column of Table I. Figure 2 shows that the co-designed cost converges to a stationary point $(J=202.8367)$. By applying the proposed co-design technique, the closed-loop system performance has been improved by $6.7 \%$ compared with the initial cost. Figures 3 and 4 also show that the closedloop system result from the co-design gives shorter settling time and less control energy.

\section{CONCLUSIONS}

This paper considered a co-design problem for a class of nonlinear constrained control systems. A novel iterative method was proposed by combining the conventional policy iteration technique and an extra optimal system design step updating the system parameters. The convergence of the proposed algorithm to a sub-optimal solution was established and the effectiveness of the proposed algorithm in improving the closed-loop system performance was demonstrated by simulation. 


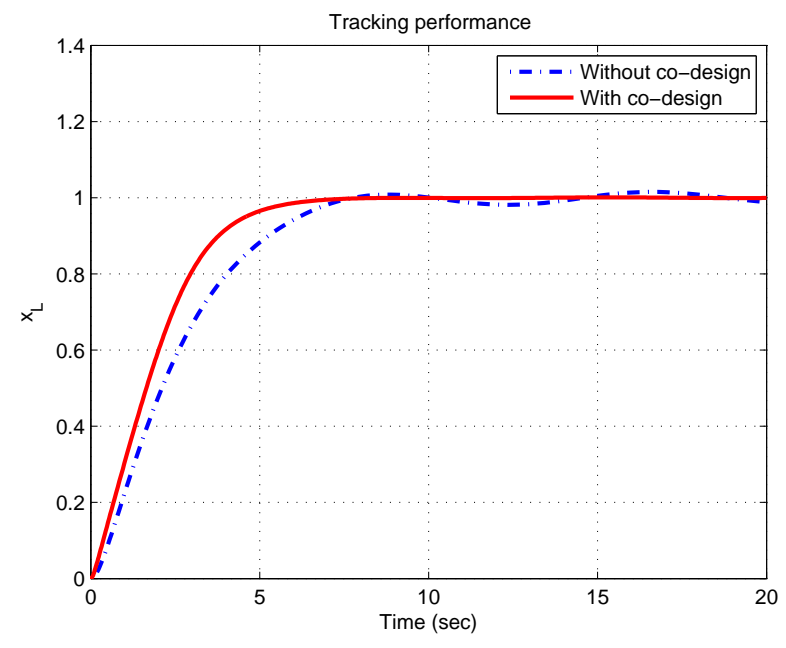

Fig. 2. Illustration of the convergence property of the proposed iterative technique, compared with the system-equivalence-based method.

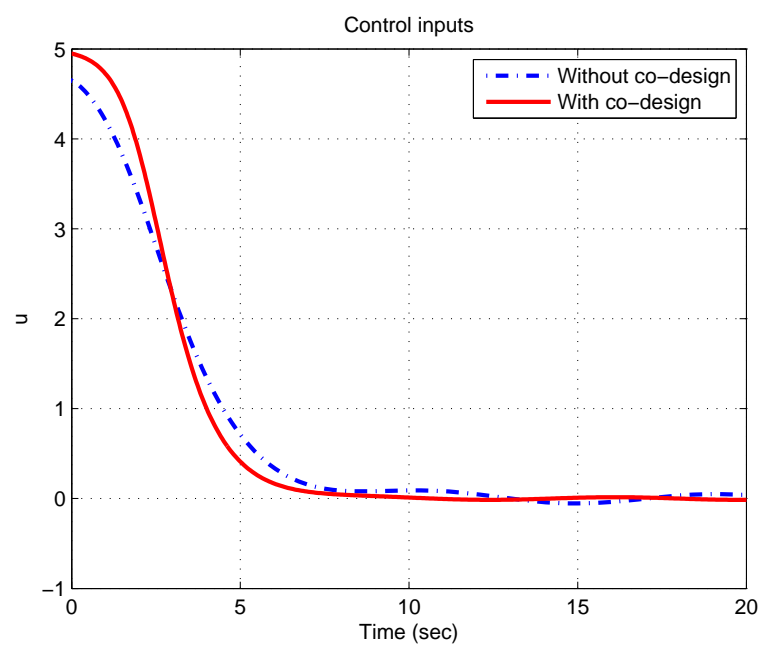

Fig. 3. Tracking performance to a step command.

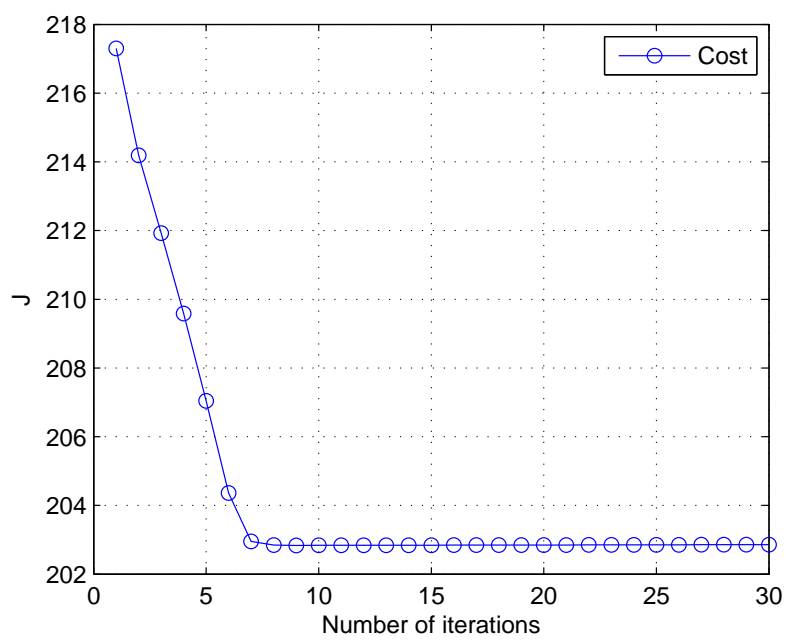

Fig. 4. Comparison of the control inputs.

\section{ACKNOWLEDGEMENTS}

Authors would like to thank Yu Jiang for constructive discussions and comments.

\section{REFERENCES}

[1] M. M. da Silva, O. Brüls, W. Desmet, and H. Van Brussel, "Integrated structure and control design for mechatronic systems with configurationdependent dynamics," Mechatronics, vol. 19, no. 6, pp. 1016-1025, 2009.

[2] J. Lu and R. E. Skelton, "Integrating structure and control design to achieve mixed $H_{2} / H_{\infty}$ performance," International Journal of Control, vol. 73, no. 16, pp. 1449-1462, 2000.

[3] K. Grigoriadis, M. Carpenter, G. Zhu, and R. Skelton, "Optimal redesign of linear systems," in Proceedings of the American Control Conference, San Francisco, CA, 1993, pp. 2680-2684.

[4] R. E. Skelton and J. H. Kim, "The optimal mix of structure redesign and active dynamic controllers," in Proceedings of the American Control Conference, Chicago, IL, 1992, pp. 2775-2779.

[5] A. L. Hale, W. Dahl, and J. Lisowski, "Optimal simultaneous structural and control design of maneuvering flexible spacecraft," Journal of Guidance, Control, and Dynamics, vol. 8, no. 1, pp. 86-93, 1985.

[6] A. Messac, "Control-structure integrated design with closed-form design metrics using physical programming," AIAA journal, vol. 36, no. 5, pp. 855-864, 1998.

[7] J. A. Reyer and P. Y. Papalambros, "Combined optimal design and control with application to an electric dc motor," Journal of Mechanical Design, vol. 124, no. 2, pp. 183-191, 2002.

[8] P. Y. Papalambros and D. J. Wilde, Principles of Optimal Design. UK: Cambridge University Press, 2000.

[9] S. F. Alyaqout, P. Y. Papalambros, and A. G. Ulsoy, "Coupling in design and robust control optimization," in European Control Conference, 2007.

[10] H. K. Fathy, J. A. Reyer, P. Y. Papalambros, and G. Ulsoy, "On the coupling between the plant and the controller optimization problems," in Proceedings of the American Control Conferences, Arlington, VA, 2001, pp. 1864-1869.

[11] R. Patil, Z. Filipi, and H. Fathy, "Computationally efficient combined design and control optimization using a coupling measure," ASME Journal of Mechanical Design, vol. 134, no. 7, p. 071008, 2012.

[12] D. L. Peters, P. Papalambros, and A. Ulsoy, "Control proxy functions for sequential design and control optimization," Journal of Mechanical Design, vol. 133, p. 091007, 2011.

[13] Y. Jiang, Y. Wang, S. A. Bortoff, and Z.-P. Jiang, "An iterative approach to the optimal co-design of linear control systems," Automatica, 2013, under review.

[14] _ "Optimal co-design of nonlinear control systems based on a modified policy iteration method," IEEE Transactions on Neural Networks and Learning Systems, 2013, under review.

[15] R. A. Howard, Dynamic Programming and Markov Processes. Cambridge, MA: MIT Press, 1960.

[16] G. N. Saridis and C.-S. G. Lee, "An approximation theory of optimal control for trainable manipulators," IEEE Transactions on Systems, Man and Cybernetics, vol. 9, no. 3, pp. 152-159, 1979.

[17] M. Abu-Khalaf and F. L. Lewis, "Nearly optimal control laws for nonlinear systems with saturating actuators using a neural network hjb approach," Automatica, vol. 41, no. 5, pp. 779-791, 2005.

[18] S. Lyashevskiy and A. U. Meyer, "Control system analysis and design upon the lyapunov method," in Proceedings of the American Control Conference, vol. 5, Seattle, WA, 1995, pp. 3219-3223.

[19] S. P. Boyd and L. Vandenberghe, Convex Optimization. Cambridge University Press, 2004.

[20] V. Shilpiekandula, S. A. Bortoff, J. C. Barnwell, and K. E. Rifai, "Load positioning in the presence of base vibrations," in Proceedings of the American Control Conference, Montreal, Canada, 2012, pp. 6282-6287. 